\title{
Initial Experience With Video-Assisted Anal Fistula Treatment in the Philippines
}

\author{
Marc Paul J. Lopez, Mark Augustine S. Onglao, Hermogenes J. Monroy III \\ Division of Colorectal Surgery, Philippine General Hospital, University of the Philippines Manila, Manila, the Philippines
}

Purpose: We determined the outcomes of patients undergoing video-assisted anal fistula treatment (VAAFT) for fistulain-ano at the Philippine General Hospital.

Methods: Twenty consecutive adult patients who underwent the VAAFT procedure from 2016-2018 were included in this investigation. Information detailing baseline demographic and clinical data, fistula type and classification, and previous surgeries were retrieved from in-hospital and operative records. Operative time, identification of the internal opening, method of internal opening closure, and occurrence of immediate postoperative complications were determined. The status of the fistula was assessed at one month, 3 months, and 6 months postoperatively based on outpatient follow-up records. The primary outcomes were healing rate and recurrence rate. Secondary outcomes were 30-day morbidity, postoperative complications, and incontinence using the Wexner score.

Results: Eighteen patients (90\%) had a preoperative diagnosis of complex fistula, and 13 patients (65\%) had undergone a previous fistula surgery. Primary healing rate was $55 \%$ at 1 month, $63.16 \%$ at 3 months, and $78.95 \%$ at 6 months postoperatively. Eighteen patients $(94.74 \%)$ maintained continence (Wexner score $=0$ ) at 6 months.

Conclusion: Our study results suggest that VAAFT is a safe, minimally invasive technique for treatment of anal fistula and can preserve anal sphincter function. The technique has an acceptable healing rate with minimal complications.

Keywords: Fistula; Anal; Anorectal

\section{INTRODUCTION}

Fistula-in-ano is one of the oldest and most common anorectal conditions. Despite continued research and development, fistulotomy remains the gold standard of treatment in most cases, particularly for intersphincteric and low transsphincteric types. However, postfistulotomy incontinence remains an issue [1].

In 2006, Meinero and Mori [2] introduced a minimally invasive technique in the form of video-assisted anal fistula treatment (VAAFT), centered around the following management philoso-

Received: 16 January 2020 - Accepted: 28 February 2020

Correspondence to: Mark Augustine S. Onglao, M.D.

Affiliation postal address: Philippine General Hospital, Taft Ave., Ermita,

Manila

Tel: +63-8554-8400,E-mail: maconglao@gmail.com

ORCID: https://orcid.org/0000-0003-2175-9959

(C) 2020 The Korean Society of Coloproctology

This is an open-access article distributed under the terms of the Creative Commons Attribution Non-

Commercial License (https://creativecommons.org/licenses/by-nc/4.0) which permits unrestricted non-

commercial use, distribution, and reproduction in any medium, provided the original work is properly cited. phies of fistula-in-ano: control of the internal opening, drainage of the main tract and any secondary tracts, and preservation of anal continence. Initial studies on this method had promising results, with a success rate of $67 \%-92 \%$. Minimal complications were reported [2].

In 2016, the Division of Colorectal Surgery at the Philippine General Hospital (PGH) obtained equipment enabling use of the VAAFT procedure (Karl Storz, Tuttlingen, Germany). At the time of this writing, the PGH is the only institution in the country to have the equipment for and experience conducting VAAFT. Expertise in performing the procedure is in progress, and it will be worthwhile to review our findings and compare our results with those in the published literature.

We evaluated the clinical outcomes of VAAFT through the early experience of a single tertiary hospital in the Philippines.

\section{MATERIALS AND METHODS}

\section{Study population}

All adult patients undergoing the VAAFT procedure (composed 
of a diagnostic phase as described by Meinero and an operative phase for control of the internal opening) [2] at the PGH from 2016-2018 were included in the study. Patients in whom VAAFT was initially planned or attempted but was converted to a different surgical procedure were excluded from long-term follow-up and data analysis.

\section{Surgical technique}

VAAFT was performed with a kit comprised of fistuloscope, anal retractor, unipolar electrode, grasper, and endobrush (Fig. 1). The fistuloscope had an $8^{\circ}$ angled eyepiece and was equipped with both an optical channel and a working channel, which doubled as an irrigation conduit. The scope diameter was $3.3 \mathrm{~mm} \times 4.7 \mathrm{~mm}$, and its operative length was $18 \mathrm{~cm}$. VAAFT was performed in 2 phases: a diagnostic phase and an operative phase [2].

The diagnostic phase was performed to locate the internal opening and identify any secondary tracts. The fistuloscope was passed through the external opening while filled with a saline-mannitol solution. The solution facilitates opening of the tract and prevents tissue edema. The fistuloscope was advanced along the tract, straightening it in the process. The internal opening was confirmed by advancement of the tip of the scope. The internal opening is often very narrow, and the location of the orifice can only be identified by observing the light from the fistuloscope visible behind the rectal mucosa (Fig. 2A). Two or 3 sutures were placed at opposite points of the margin of the internal opening to isolate but not close this opening [2].

The operative phase was then performed to curette the fistula tract and close the internal opening. First, an electrode was used to electrodessicate the fistula tract under direct visualization. The electrode was gradually passed from the external opening to the

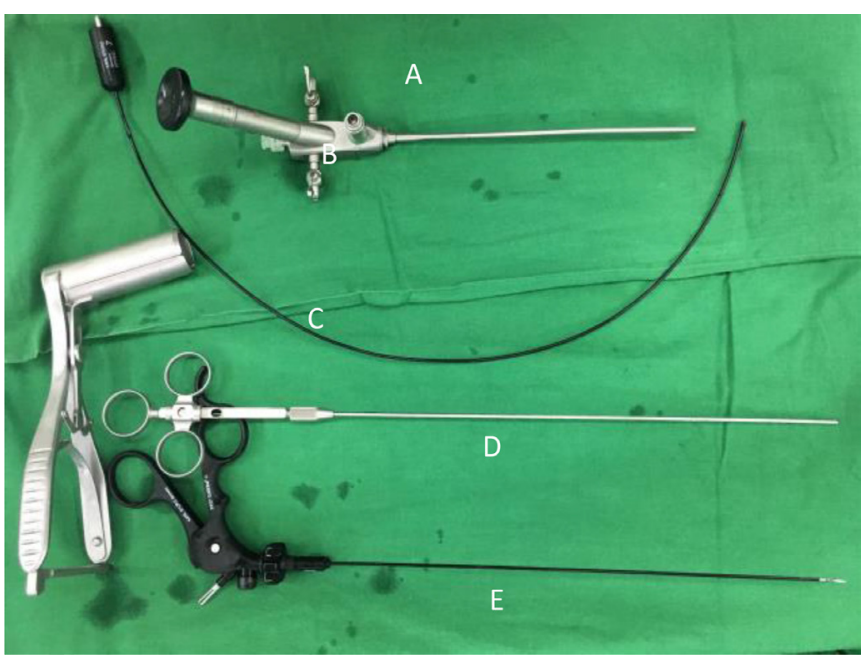

Fig. 1. Video-assisted anal fistula treatment equipment. (A) Fistuloscope. (B) Unipolar electrode. (C) Anal retractor. (D) Endobrush. (E) Grasper. internal opening, cauterizing all fragments of the material adhering to the fistula wall until the pink fistula tract became brown in color (Fig. 2B, C). Continuing under direct visualization, any necrotic material was removed using an endobrush (Fig. 2D) or a Volkmann spoon in cases where the fistula was straight. A continuous jet of the irrigation solution ensured that all waste material was eliminated into the rectum through the internal opening that had been isolated [2].

After thorough cleaning of the fistula tract, the internal opening was closed. The original technique described by Meinero provides 3 methods of internal opening closure: suturing, transanal stapler, or a mucosal flap. The decision to perform a flap was made if there was sufficient scarring at the internal opening to prevent adequate closure of the tissue via stapling or suturing. Other surgeons have described additional techniques of internal opening control, such as ligation of an intersphincteric fistula tract (LIFT) or seton placement $[3,4]$. Due to the unavailability of a transanal stapler and reservations to use of a mucosal flap as a bail-out technique in case of multiple failed surgeries, we used suturing, LIFT, fistulotomy, or seton placement.

\section{Data collection and follow-up}

Clinical records of patients who underwent VAAFT from 20162018 were reviewed. Baseline demographic and clinical data, fistula type (simple vs. complex) and classification (using the Parks classification system), and number of previous surgeries were retrieved from in-hospital and operative records. Operative time, identification of the internal opening, method of internal opening closure, and occurrence of immediate postoperative complications (e.g., bleeding, cellulitis, scrotal edema, and urinary retention) were determined. The status of the fistula was assessed at 1, 3, and 6 months postoperatively using outpatient follow-up records.

The primary outcome measured was healing rate. "Healing" was defined as the absence of perianal discharge and complete epithelialization of the perianal wound/external opening. In patients with multiple tracts, healing was considered only if all tracts were fully epithelialized. "Nonhealing" was defined as persistence of perianal discharge or nonepithelialization of the wound 3 months after surgery. Recurrence rate and time to recurrence also were determined. Recurrence was defined as relapse of perianal discharge or opening of the perianal wound after initial healing.

Secondary outcomes measured were 30-day morbidity and presence of fecal incontinence. Incontinence was defined as inability to control flatus, liquid stools, or solid stools after surgery and was assessed using the Wexner score (Table 1) [5]. The healing rate of each method of internal opening closure was measured.

\section{Ethical considerations}

The study was conducted in accordance with the guidelines of the Declaration of Helsinki, the ICH-GCP principles, the provisions of the National Ethical Guidelines for Health and Health-related 


\section{$\begin{aligned} \text { Annals of } & \text { Initial Experience With Vi } \\ \text { Coloproctology } & \text { Marc Paul J. Lopez, et al. }\end{aligned}$}
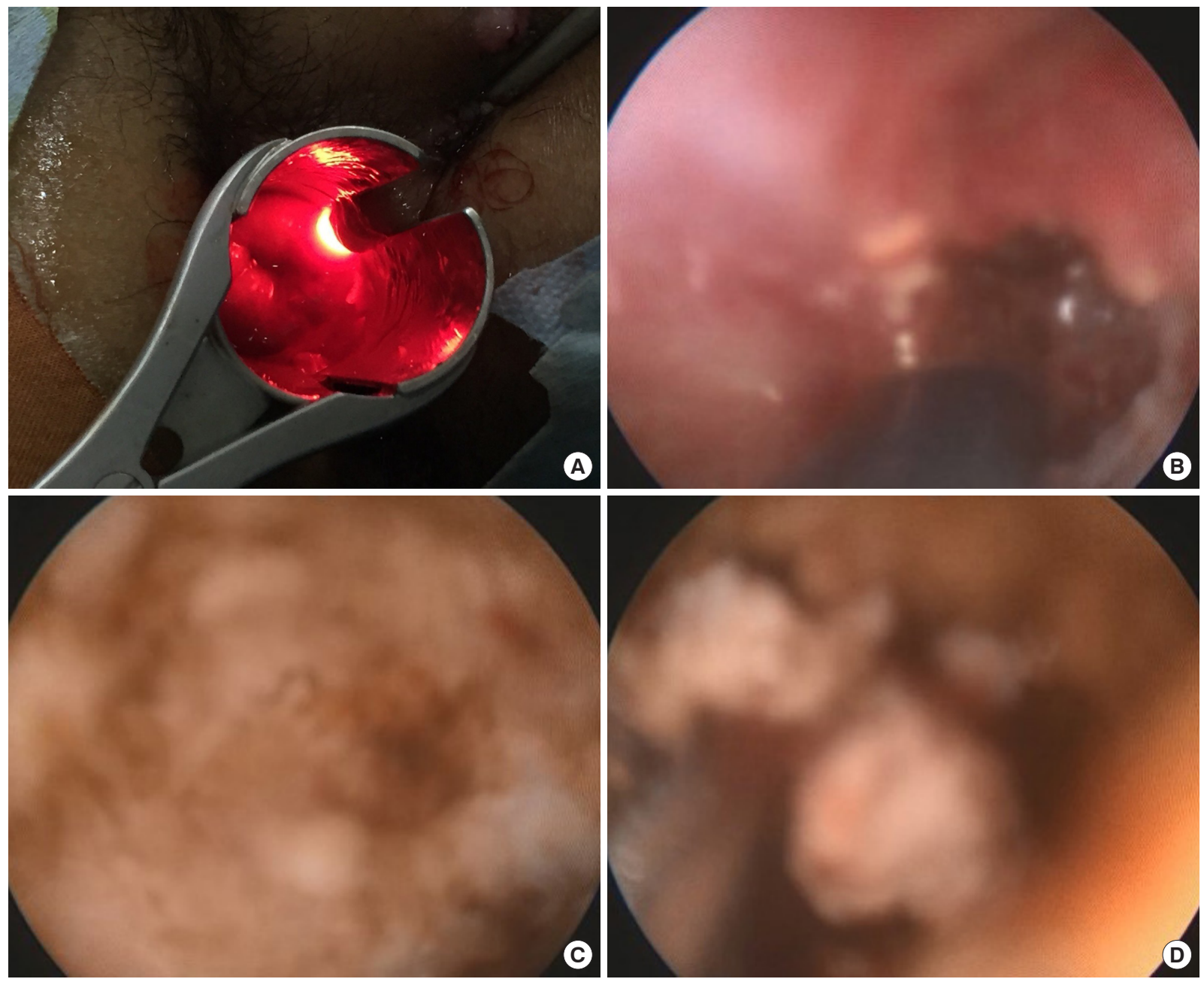

Fig. 2. (A) Light from the fistuloscope as seen through the rectal mucosa, indicating the location of the internal opening. (B) Electrodessication with a unipolar electrode. (C) Fistula tract color changed from pink to brown, indicating complete electrodessication. (D) Removal of debris with an endobrush.

Table 1. Jorge-Wexner Incontinence Score

\begin{tabular}{lccccc}
\hline \multirow{2}{*}{ Type of incontinence } & \multicolumn{5}{c}{ Frequency } \\
\cline { 2 - 6 } & Never & Rarely & Sometimes & Usually & Always \\
\hline Solid & 0 & 1 & 2 & 3 & 4 \\
Liquid & 0 & 1 & 2 & 3 & 4 \\
Gas & 0 & 1 & 2 & 3 & 4 \\
Wears pad & 0 & 1 & 2 & 3 & 4 \\
Lifestyle alteration & 0 & 1 & 2 & 3 & 4 \\
\hline
\end{tabular}

Never, 0; Rarely, <1/month; Sometimes, <1/week, $>1$ /month; Usually, $<1 /$ day, $>1 /$ wk; Always, $>1 /$ day.

Full continence total 0; Complete incontinence total 20.

Research of 2017, and the Data Privacy Act of 2012 (RA 10173). The study was reviewed and approved by the University of the
Philippines Manila Research Ethics Board (UPM-REB). As the proposed study only involved review of patient records and collection of routinely collected, nonsensitive data, a waiver of consent was approved by the UPM-REB.

\section{Statistical analysis}

Descriptive statistics were used to summarize the demographic and clinical characteristics of the patients. Frequency and proportion were used for categorical variables, while mean and standard deviation were applied to normally distributed continuous variables. Cochran Q test was used to determine the difference in parameters of the patients from baseline to the sixth-month followup visit. The Shapiro-Wilk test was used to assess the normality of continuous variables. Missing variables were neither replaced nor estimated. Stata 13.1 (StataCorp LP., College Station, TX, USA) was used for data analysis. 


\section{RESULTS}

Twenty patients who underwent the VAAFT procedure were included in this study. The baseline demographic and clinical characteristics of the patients are listed in Table 2 . There were 18 males and 2 females, with a mean age of 38.2 years (range, $18-56$ years). Seven patients (35\%) had a newly diagnosed fistula with no history of previous procedures. The other 13 patients $(65 \%)$ had undergone previous fistula surgery: 9 fistulotomies/fistulectomies, 2 seton placements, and 2 LIFT procedures. Eighteen patients had a preoperative diagnosis of complex fistula due to recurrent fistulas, having presence of multiple external openings, or a fistula that involved a significant amount of the anal sphincter.

All patients underwent the VAAFT procedure as described. The results of the surgery are summarized in Table 3 . Mean operative time was 90.8 minutes (range, $45-130$ minutes). The types of fistulas based on the Parks Classification are shown in Table 2. Most (9 of 20,45\%) had high transsphincteric fistulas. The internal opening was located in the diagnostic phase in $75 \%$ of patients (15 of 20 patients). Of these 15 patients, the internal opening was controlled by suturing ( 4 patients, $26.7 \%$ ), seton placement ( $6 \mathrm{pa}-$ tients, $40.0 \%$ ), or fistulotomy (5 patients, 33.3\%). We did not use the LIFT procedure for patients in this study but have adopted the procedure in subsequent cases of VAAFT.

Two patients experienced perioperative complications: 1 patient had a chipped tooth during intubation for general anesthesia, and another developed postoperative rashes and pruritus secondary to an adverse drug reaction to analgesics that resolved with antihistamines. There were no complications related to the surgery it-

Table 2. Demographic profile of patients undergoing VAAFT

\begin{tabular}{lc}
\hline Variable & Value \\
\hline Age (yr) & $38.2 \pm 9.95$ \\
Sex & $18(90)$ \\
Male & $2(10)$ \\
Female & \\
Fistula type & $2(10)$ \\
Simple & $18(90)$ \\
Complex & \\
Recurrent fistula after a previous procedure & $13(65)$ \\
Recurrent fistula & $7(35)$ \\
Newly diagnosed fistula & \\
Previous surgery (n=13) & $9(69.23)$ \\
Fistulotomy/Fistulectomy & $2(15.38)$ \\
Seton & $2(15.38)$ \\
\hline LIFT &
\end{tabular}

Values are presented as mean \pm standard deviation or number (\%).

VAAFT, video-assisted anal fistula treatment; LIFT, ligation of an intersphincteric fistula tract. self. All patients were discharged within 2 days of surgery.

Nineteen patients completed the 6-month follow-up. One patient was lost to follow-up and failed to return to the outpatient department after surgery. Mean follow-up duration for all patients in the study was 19.6 months (range, 6-30 months). Primary healing rate was $55 \%$ at 1 month, $63.16 \%$ at 3 months, and $78.95 \%$ at 6 months postoperatively. The outcomes of the patients at 1,3 and 6 months follow-up are summarized in Table 4.

One patient experienced recurrence after initial epithelialization of a wound that occurred after 3 months of follow-up. The patient

Table 3. Perioperative outcomes of video-assisted anal fistula treatment $(\mathrm{n}=20)$

\begin{tabular}{lc}
\hline Variable & Value \\
\hline Total time & $90.8 \pm 26.09$ \\
Internal opening located & \\
Yes & $15(75.0)$ \\
No & $5(25.0)$ \\
Fistula classification & \\
Intersphincteric & $1(5.0)$ \\
Low transsphincteric & $6(30.0)$ \\
High transsphincteric & $9(45.0)$ \\
Supra-sphincteric & $4(20.0)$ \\
Method for closure of the internal opening (n=15) & \\
Suturing & $4(26.7)$ \\
Seton & $6(40.0)$ \\
Fistulotomy & $5(33.3)$ \\
Perioperative complications & $2(10.0)$ \\
Rash/pruritus & $1(5.0)$ \\
Chipped tooth & $1(5.0)$ \\
Postoperative length of stay (day) & \\
1 & $8(40.0)$ \\
\hline
\end{tabular}

Values are presented as mean \pm standard deviation or number (\%).

Table 4. Outcomes of video-assisted anal fistula treatment at 1, 3, and 6 months of follow-up

\begin{tabular}{lccc}
\hline Variable & 1 Month & 3 Months & 6 Months \\
\hline Healing & $11(55.0)$ & $12(63.16)$ & $15(78.95)$ \\
Recurrence & $0(0)$ & $1(5.26)$ & $1(5.26)$ \\
Wexner score & & & \\
0 & $17(85.0)$ & $17(89.47)$ & $18(94.74)$ \\
4 & $1(5.0)$ & $1(5.26)$ & $0(0)$ \\
6 & $2(10.0)$ & $1(5.26)$ & $1(5.26)$ \\
Total follow-up duration (mo) & $19.68 \pm 8.04$ & - & - \\
\hline
\end{tabular}

Values are presented as number (\%) or mean \pm standard deviation. 


\section{Coloproctology $\quad$ Marc Paul J. Lopez, et al.}

Table 5. Outcomes of VAAFT based on technique for closure of the internal opening

\begin{tabular}{lcccc}
\hline & Not identified $(\mathrm{n}=5)$ & Suturing $(\mathrm{n}=4)$ & Fistulotomy $(\mathrm{n}=5)$ & Cutting seton $(\mathrm{n}=6)$ \\
\hline Healed after 6 months & $4(80.0)$ & $2(50.0)$ & $5(100)$ & $4(66.7)$ \\
\hline
\end{tabular}

Values are presented as number (\%).

VAAFT, video-assisted anal fistula treatment.

has declined further treatment. Eighteen patients (94.74\%) maintained continence (Wexner score $=0$ ) at 6 months postoperatively. One patient had a Wexner score of 6 at the 6-month follow-up visit. This patient complained of incontinence to flatus and liquid stool that occurred less than once per week. This patient's symptoms were addressed with stool bulking agents. The healing rate of each method of internal opening closure is shown in Table 5. The healing rate in patients where the internal opening was not identified but the tract was electrodessicated and curetted was $80 \%$. Suturing of the internal opening had a 50\% healing rate. All patients who underwent fistulotomy had healed after 6 months. Of those who underwent placement of a cutting seton, $66.67 \%$ had healed fistulas at 6 months after 2 to 3 sessions of seton tightening.

\section{DISCUSSION}

A fistula-in-ano is an epithelialized communication between the rectum or anal canal and the perianal region that most commonly occurs following a cryptoglandular infection [1]. Surgical treatment of fistula-in-ano remains formidable. Numerous techniques have been developed, but no single technique has been found to be appropriate for every case.

For simple fistula, where $<30 \%$ of the external sphincter is involved, a fistulotomy may be most appropriate. The healing rate of this technique ranges from $93 \%-100 \%$. Because the sphincters are inevitably transected in this procedure, minor incontinence is reported in $11.5 \%-20 \%$ of cases [6-9]. For complex fistulas, however, treatment is more difficult and carries a higher recurrence rate and greater potential of external sphincter damage [10]. Complex fistulas-in-ano include high transsphincteric, suprasphincteric, and extrasphincteric fistulas (tract crosses 30\%-50\% of the external sphincter); anterior fistulas in females; fistulas with multiple tracts; or patients with pre-existing incontinence, a history of local irradiation, or Crohn disease [11].

To address the problem of high recurrence and incontinence rates in these fistulas, many techniques have been developed, including seton placement, endorectal advancement flap, fibrin glue, anal fistula plug, LIFT and, most recently, VAAFT [2, 12-16].

The recognized advantages of VAAFT are as follows: (1) It provides accurate identification of the internal opening and any secondary tracts. (2) It allows complete destruction of the tract under direct visualization. (3) It is a minimally invasive technique that does not cut any sphincter and leaves a small perianal wound, which allows for to faster recovery and less postoperative pain [17].

The first advantage of VAAFT is that it allows surgeons to deter- mine fistula anatomy under direct visualization. Historically, fistula anatomy and internal openings have been defined through "blind" probing techniques or using an infusion of hydrogen peroxide or methylene blue. However, probing can result in accidental creation of iatrogenic tracts that may lead to nonhealing. Newer imaging techniques include magnetic resonance imaging (MRI) and endoanal ultrasonography (EAUS). Although both are very sensitive modalities, MRI is expensive, and EAUS is not universally available and is operator dependent [18]. VAAFT well defines the fistula anatomy by exploring the main tract and its secondary branches under direct visualization. Emile et al. [19] noted in their review that the internal opening was identified in $85.7 \%$ of patients, and secondary tracts were identified in $25.3 \%$ of cases. Our study was able to locate the internal opening in $75 \%$ of cases. However, the accuracy of the findings from the diagnostic phase of VAAFT was not compared to any preoperative imaging. As a result, the sensitivity and specificity of VAAFT in determining fistula anatomy remain unknown and should be explored in further studies.

Complete destruction of the tracts is possible under direct visualization with VAAFT. The initial results published by Meinero and Mori [2] showed an $87.1 \%$ healing rate and noted no complications or incontinence. Other surgeons have performed this same procedure and reported similar results. A systematic review by Garg and Singh [20] reported a $76 \%$ healing rate $(95 \%$ confidence interval [CI], 68.1\%-83.9\%). Emile et al. [19] published a systematic review showing a weighted mean recurrence rate of $17.7 \%$ (95\% CI, $11.8 \%-23.7 \%$ ) for VAAFT with a median follow-up duration of 9 months (range, 3.2-34 months). This outcome is comparable to the reported recurrence rates for LIFT (24.6\%) and for endorectal advancement flaps (21\%) [20]. Our series had a healing rate of 78.95\% and a recurrence rate of $5.26 \%$ at the 6-month follow-up visit.

VAAFT is also minimally invasive and sphincter-sparing. Furthermore, the technique is fast and can be performed in an ambulatory care setting. Postoperative pain is minimal to none. Therefore, patients are able to return to work earlier than they can if they undergo other procedures. The technique avoids large perianal wounds that are painful and more difficult to nurse. Reoperations can be performed using the same technique in cases of recurrence. In the literature, mean operative time for VAAFT was 44.7 minutes (95\% CI, 38.3-51.2). Hospital stay ranged from 1.02-4.1 days. Return to work ranged from 1-11 days. Mean complication rate was $4.8 \%$ with no deterioration in sphincter function [20].

Our study had a longer mean operative time of $90.8 \pm 26.09$ 
minutes. This length may be attributed to a report of our initial experience of 20 cases, during which we were on a learning curve. Although some studies have recognized that operative time and recurrence rate improve with experience with the VAAFT technique, no study has been published regarding the number of cases required to become proficient in performing this surgery. Large variation in our operative times may be due to the variety of methods used to close the internal opening.

Hospital stay was shorter in our study, at 1-2 days. As we continue to master this technique, operative time and hospital stay duration may be shortened further. We are also investigating the possibility of performing VAAFT as a day surgery, similarly to other fistula operations at our institution.

The complication rate in our series was $10 \%$, but we found only minor complications that were not directly related to the procedure itself. Most patients (94.74\%) had full continence after 6 months based on the Wexner score. One patient (5.26\%) had a Wexner score of 6 (mild incontinence) and underwent cutting seton placement to control his internal opening, with subsequent tightening after 2 weeks.

The most appropriate method of internal opening closure in VAAFT needs to be determined. In their systematic review, Emile et al. [19] reported the success rate of the methods of internal opening closure with the following results. The recurrence rate was $15.3 \%$ (69 of 449) after closure with a stapler, $17.7 \%$ (20 of 113 ) after closure with sutures, and $25 \%$ (7 of 28) after closure using an advancement flap. As expected, all 3 patients in which the internal opening was left open developed recurrence [19]. This trend was not observed in our study, where $80 \%$ of patients with a remaining internal opening demonstrating full healing. It is uncertain whether these patients had true fistulas or persistent sinuses. Longer follow-up periods are needed to determine if such fistulas will recur. Suturing of the internal opening produced worse outcomes, with a $50 \%$ healing rate. All patients who underwent fistulotomy had healed at 6 months postoperatively. Of those who underwent cutting seton placement, $66.67 \%$ had healed fistulas at 6 months. This result is comparable to the published outcomes of other techniques. It is still unclear whether the success of VAAFT is due to the method of internal opening closure or complete obliteration and cauterization of the fistula and secondary tracts under its direct visualization. Other methods of internal opening closure, such as over-the-scope clip (OTSC, Ovesco Endoscopy AG, Tubingen, Germany) closure or stapled closure, were not explored due to unavailability of both methods in our country. More studies are needed to compare these different techniques.

At present, it appears that VAAFT offers more value during the diagnostic phase. Accurate delineation of tract anatomy and any secondary tracts and accurate location of the internal opening provide essential information to guide surgical decision-making. However, the best option for controlling the internal opening during the VAAFT procedure needs further investigation. The rec- ommended methods of stapling or using an OTSC are costly and are not readily available in low-resource countries. Suturing of the internal opening, although inexpensive and simple, has higher recurrence rates, as demonstrated in our study and in other reports. Lastly, a mucosal advancement flap is technically demanding and carries a minor risk for serious complications, such as flap breakdown or infection, and may not be suitable for all patients. Other methods, including fistulotomy and seton placement, negate the sphincter-sparing advantage of the VAAFT technique.

Limitations of our study include its small sample size and absence of long-term follow-up data. The method of internal opening closure was not standardized and was left to the surgeon's discretion. This variation in closure may have confounded the results in our study because healing in these patients could be attributed primarily to the technique used to control the internal opening and not the VAAFT technique itself.

Nevertheless, our study results suggest that VAAFT is a safe and minimally invasive technique for treatment of anal fistula and can preserve anal sphincter function. It has an acceptable healing rate with minimal complications and a fast recovery time.

\section{CONFLICT OF INTEREST}

No potential conflict of interest relevant to this article was reported.

\section{REFERENCES}

1. Jiang HH, Liu HL, Li Z, Xiao YH, Li AJ, Chang Y, et al. Video-assisted anal fistula treatment (VAAFT) for complex anal fistula: a preliminary evaluation in China. Med Sci Monit 2017;23:2065-71.

2. Meinero P, Mori L. Video-assisted anal fistula treatment (VAAFT): a novel sphincter-saving procedure for treating complex anal fistulas. Tech Coloproctol 2011;15:417-22.

3. Romaniszyn M, Walega PJ. Are two better than one? VALIFT: video-assisted ligation of the intersphincteric fistula tract-a combination of two minimally invasive techniques for treatment of transsphincteric perianal fistulas. Tech Coloproctol 2019;23:273-6.

4. Tozer P, Sahnan K, Adegbola S, Shaikh S, Lung P. Video-assisted anal fistula treatment (VAAFT) assisted seton placement - a video vignette. Colorectal Dis 2019;21:1462.

5. Jorge JM, Wexner SD. Etiology and management of fecal incontinence. Dis Colon Rectum 1993;36:77-97.

6. Oliver I, Lacueva FJ, Pérez Vicente F, Arroyo A, Ferrer R, Cansado $\mathrm{P}$, et al. Randomized clinical trial comparing simple drainage of anorectal abscess with and without fistula track treatment. Int J Colorectal Dis 2003;18:107-10.

7. Hall JF, Bordeianou L, Hyman N, Read T, Bartus C, Schoetz D, et al. Outcomes after operations for anal fistula: results of a prospective, multicenter, regional study. Dis Colon Rectum 2014;57:1304-8.

8. Atkin GK, Martins J, Tozer P, Ranchod P, Phillips RK. For many high anal fistulas, lay open is still a good option. Tech Coloproc- 


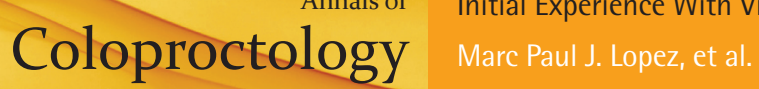

tol 2011;15:143-50.

9. Tozer P, Sala S, Cianci V, Kalmar K, Atkin GK, Rahbour G, et al. Fistulotomy in the tertiary setting can achieve high rates of fistula cure with an acceptable risk of deterioration in continence. J Gastrointest Surg 2013;17:1960-5.

10. Kochhar G, Saha S, Andley M, Kumar A, Saurabh G, Pusuluri R, et al. Video-assisted anal fistula treatment. JSLS 2014;18(3). pii: e2014.00127. https://doi.org/10.4293/JSLS.2014.00127.

11. Narang SK, Keogh K, Alam NN, Pathak S, Daniels IR, Smart NJ. A systematic review of new treatments for cryptoglandular fistula in ano. Surgeon 2017;15:30-9.

12. Haim N, Neufeld D, Ziv Y, Tulchinsky H, Koller M, Khaikin M, et al. Long-term results of fibrin glue treatment for cryptogenic perianal fistulas: a multicenter study. Dis Colon Rectum 2011;54:127983.

13. Lentner A, Wienert V. Long-term, indwelling setons for low transsphincteric and intersphincteric anal fistulas. Experience with 108 cases. Dis Colon Rectum 1996;39:1097-101.

14. Abbas MA, Lemus-Rangel R, Hamadani A. Long-term outcome of endorectal advancement flap for complex anorectal fistulae. Am Surg 2008;74:921-4.

15. Garg P, Song J, Bhatia A, Kalia H, Menon GR. The efficacy of anal fistula plug in fistula-in-ano: a systematic review. Colorectal Dis 2010;12:965-70.

16. Hong KD, Kang S, Kalaskar S, Wexner SD. Ligation of intersphincteric fistula tract (LIFT) to treat anal fistula: systematic review and meta-analysis. Tech Coloproctol 2014;18:685-91.

17. Sheikh P, Baakza A. Management of fistula-in-ano-the current evidence. Indian J Surg 2014;76:482-6.

18. Wałęga P, Romaniszyn M, Nowak W. VAAFT: a new minimally invasive method in the diagnostics and treatment of anal fistulas-initial results. Pol Przegl Chir 2014;86:7-10.

19. Emile SH, Elfeki H, Shalaby M, Sakr A. A Systematic review and meta-analysis of the efficacy and safety of video-assisted anal fistula treatment (VAAFT). Surg Endosc 2018;32:2084-93.

20. Garg P, Singh P. Video-assisted anal fistula treatment (VAAFT) in cryptoglandular fistula-in-ano: a systematic review and proportional meta-analysis. Int J Surg 2017;46:85-91. 